\title{
Application in Military Equipment of Our Country Based on TENA Architecture
}

\author{
Z.R. WU \& M.Q. CHEN \& W.N. CUI \\ Department of Information Engineering, Academy of Armored Forces Engineering, Beijing, China
}

Keywords: Simulation System; TENA; Architecture

\begin{abstract}
Currently, there are many independent and closed "chimney" simulation systems in some range training systems, which is not only a waste of resources but incompatible with other systems well. In view of this situation, the paper focused on using of the testing and training architecture (TENA) that US developed to make improvements. According to the characteristics of TENA's architecture, the paper summarizes the framework to adapt the domestic simulation systems. Finally, the conclusion shows that it can indeed improves the performance of the system using TENA in the domestic simulation systems.
\end{abstract}

\section{INTRODUCTION}

Architecture is a part of universal project. If it has not overall architecture, any new developed system will become an individual which a little relation with other systems. Thus it will produce many chimney systems. Architecture is the foundation and key of the virtual systems. Currently, the virtual system architecture has been gradually moving from a centralized, closed to a distributed, open, interactive development. Making more efficient to use of range resources, improve joint testing and training capabilities to minimize the costs of range operation in future.

The US military launched a FI2010 (Foundation Initiative 2010) project, the goal is to build architecture and technical support that logic range provided to meet the military needs of test and training in the coming decades. FI2010 engineering group draw a lot of simulation architecture which defines the TENA. The purpose is to give the US military test and training ranges and their users to provide public architecture, and to make the variety of geographically distributed, pilot training resources on separate functions (including equipment platform, range instrumentation, simulation systems and a variety of commands and control systems, etc.) to combined to form an integrated environment to realistic, economical and efficient way to complete the joint testing and training mission NCW required .

\section{TENA RESEARCH}

TENA (Test and Training Enabling Architecture) is proposed by US military based on HLA (High Level Architecture) and makes extension and provides testing and training required more specific functions. it can be considered a superset of HLA. Its purpose is to meet the specific needs of testing and training areas.

TENA is designed to use a large-scale, distributed, real-time integrated environment for remote testing and simulation, including the complete test, training, simulation and high-performance computing technology which makes equipment in different areas to use a unified architecture. Now range building has many cases of "chimney" systems. They are suitable for different sensors, networks, protocols, hardware and software. If you want to use the current range resources for military training more efficiently, this situation must be changed. Future testing and training systems need to integrate complex systems range.

TENA overcomes the situation that builds "chimney" system and achieves ranges resources interoperability, reuse, combined which makes between the simulation of weapons and troops distributed around the world to affect each other to form a logic range of testing and training. 


\subsection{The functional components of TENA}

By examining the various systems used TENA mainly describes the operating range of a logical concept to guide the planning, establishment and use of a "logical range". From these systems functions will be divided into six categories. they indicate the type of the various systems used in today's range, then determine systems that the logical range must support . Logical Range that six types of system components is shown in Figure1.

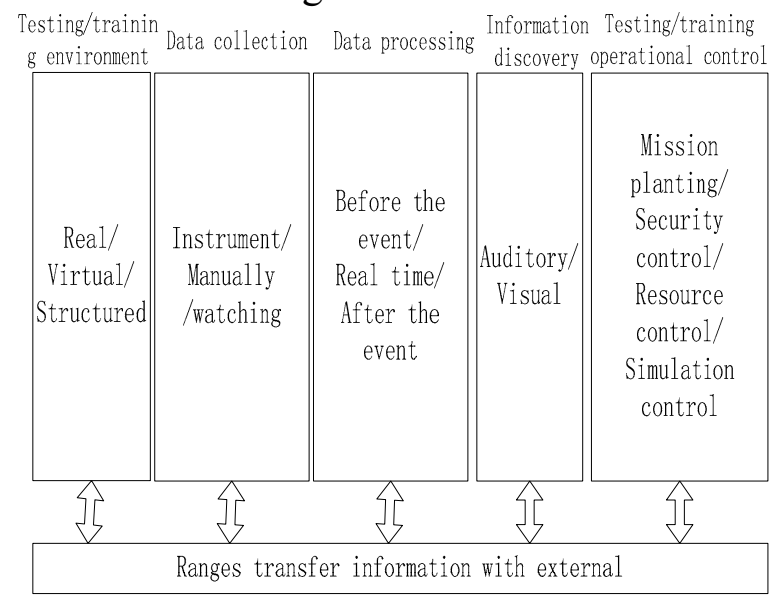

Figure 1. Range system functional decomposition

\subsection{TENA's Driving Requirements}

Architecture is the bridge of requirements and design of many important systems. One of the most important and most abstract is the system drive Requirements. TENA's driving requirements are derived from a detailed analysis of DoD-supplied source documents, as well as feedback from the

range community itself. Divided into two categories:operational driving requirements and technical driving requirements.

(1) the operational driving requirements

Include: Range event supports logic implementations of range throughout the entire cycle. Foundation for the testing and training network-centric warfare. Support the rapid development deployment and application of the logical range. Support the fast integration of model and simulation. Simulation-based and promote realization of the concept of joint distributed engineering plant. Support the smooth deployment of applications to various ranges. Meeting the performance requirements of various systems of universal range.

(2) technical driving requirements

The most important technical driving requirements of TENA is interoperability, reuse, and composability. Interoperability is that the components, applications or systems and can work with other elements independently. Its focus is on those elements of public things. For TENA, the most important interoperability is the level of semantic, which is built on the foundation consisting of a common language and context for communication. Reuse is the ability to use a software element in a context which it was not originally intended, and is focused on the multiple uses of a single element. Composability is the ability that lots of reusable, interoperable software elements can be combined according to different needs of different characteristics into the system.

\section{TENA'S SYSTEM ARCHITECTURE}

Currently, TENA system architecture based on TENA's driving requirements design defines five basic softwares, as shown in Figure 2. 


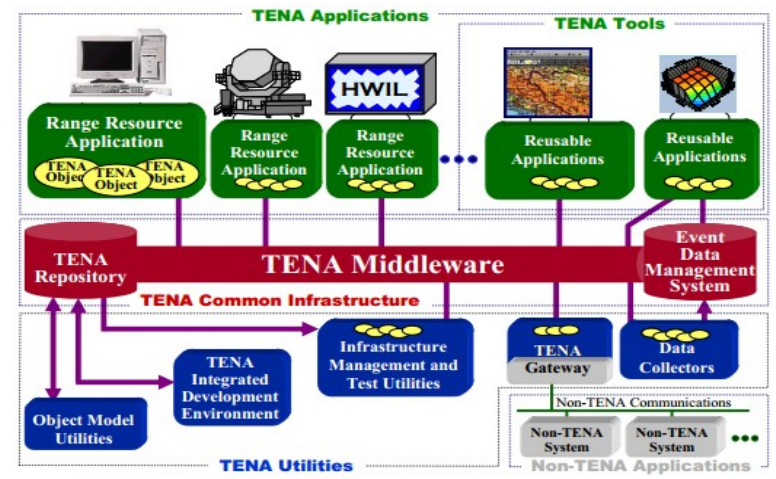

Figure 2. TENA system architecture diagram overview

(1) TENA applications(green), including range resources applications and TENA tools. Range Resources applications is simulation system, Range Resources and other physical facilities that is compatible with TENA. They are the heart of any logical range. TENA tools are generally reusable TENA applications and some of the key softwares. They help to promote the management for the whole incident lifecycle logical range.

(2) Non-TENA applications(gray) are required simulation systems, range of resources and facilities but with some TENA incompatible. they interoperate with the TENA applications by the gateway.

(3)The TENA public infrastructures(red) including TENA Middleware, TENA warehouses, TENA logical range data archives, are core part of TENA.

(4) The TENA object models(yellow) are the common language communication between all tools and range resources. Object model that a logical range uses is called "logical range object model" which is defined on the basis of TENA object model and may include the TENA standard object definitions, also containing non-standard definition.

(5) TENA utilities(blue) designed for the logic range to use TENA and corresponding management. These programs can make resources of distributed in different range integration together, and its effective management and optimize the public infrastructure of TENA.

\section{TENA'S TECHNOLOGY SYSTEM}

\subsection{The TENA's technology status}

Since TENA was produced, it continued to complete and mature. Mainly include:

(1) The definition of TENA's meta-model stabilizes. The stabilization of meta-model is in favor of the research and applications of TENA, especially for the implementation of the definition of TENA Object Model and the TENA Middleware.

(2) The TENA warehouse was based on Web. Providing the object model and the basic realization of many object definitions, including the standard TENA radar object model and TENAGPS object model.

(3) The performance of TENA Middleware is continuously improved. Fixing defects and reducing the number of IP multicast threads. Optimizing pointer operations and improving the operating speed. Supporting local classes downcast.

(4)The tools system that TENA support continuously improve.

4.2 The TENA communication mechanism

The communication mechanism of TENA Middleware is a combination of two powerful of concepts that include distributed object and data-centric publishing-ordering mechanism. Traditional object-oriented distributed systems do not support one-to-many modes of transmission of user data. The traditional publishing ordering system does not support abstract objects with methods in the interface, such as HLA (High Level Architecture) object model template. It only provides to support for interactive classes and its parameters, object classes and attributes, without any the method, which reflects the shortcomings of the data-centric architecture. However, publishing-ordering mechanism provides good support for data communication of one-to-one and communication of publishing to ordering in favor of large-scale distributed system implementation. 
Thus, TENA comprehensives these two concepts that are distributed object computing and publishing-ordering mechanisms. The two complement each other and forming a unique communication mechanism.

At present, many systems are based on HLA architecture. In order to connect with TENA architecture requires a corresponding TENA-HLA gateway. It can be designed according to the needs of TENA-HLA gateway that has three parts: TENA management section, HLA management section and gateway management section. Gateway architecture shown in Figure 3.

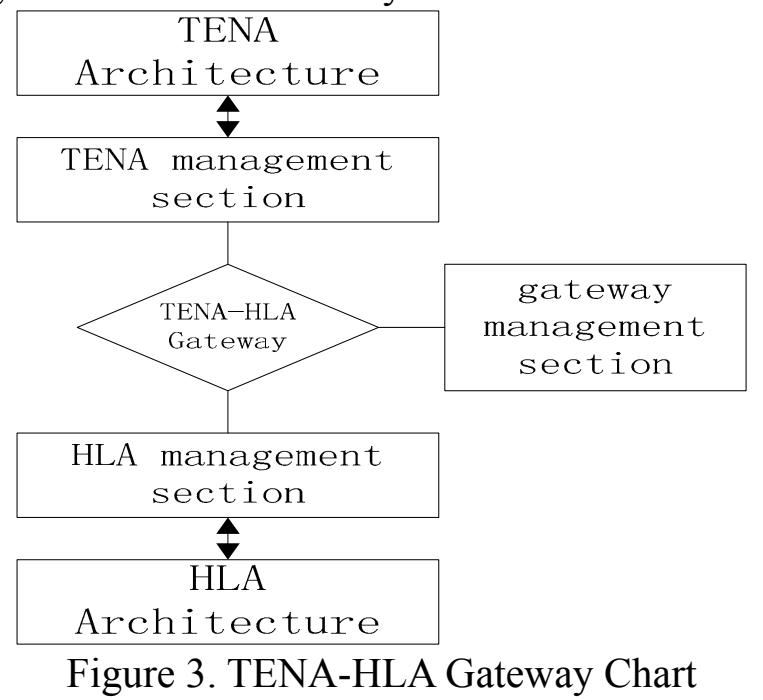

TENA management section is quite a TENA applications, including SDO, messages and data flow etc., as well as a function class with a TENA object and HLA object. HLA management section is quite an HLA federate that includes some interactive classes, RTI interface classes, as well as mapping between class names and handles. Gateway management section is responsible for starting, the whole cycling and quitting of the gateway, and the work of all part of the coordination and monitoring of the entire network.

\section{TENA APPLICATION IN CHINA}

TENA application has been nearly a decade. Testing and training in the US has been very popular. The application of TENA also makes range resources of US military to have been well integrated in the testing and training, which saves a lot of resources and also improves the efficiency of military training. Because of TENA's advantage, many countries have carried out on the basis of its to suit their military testing and training requirements.

For our country, our military resources are complex, geographical distribution widely. The technology of testing and training in the field of logic range is relatively backward. Thus causing a lot of waste and lots of manpower and financial resources. With the deepening of research in recent years, China's many agencies and units study TENA, in order to improve the current situation waste of resources and the technology behind. The outcome is very fruitful.

For now, the testing and training systems based on TENA ideology or on the basis of TENA framework generally have similar architecture. Divided into three parts: bottom layer has operating system, database management systems and other hardware components; middle layer(including: TENA and other auxiliary systems); application layer section includes some simulation applications. Frame is shown in figure 5. 


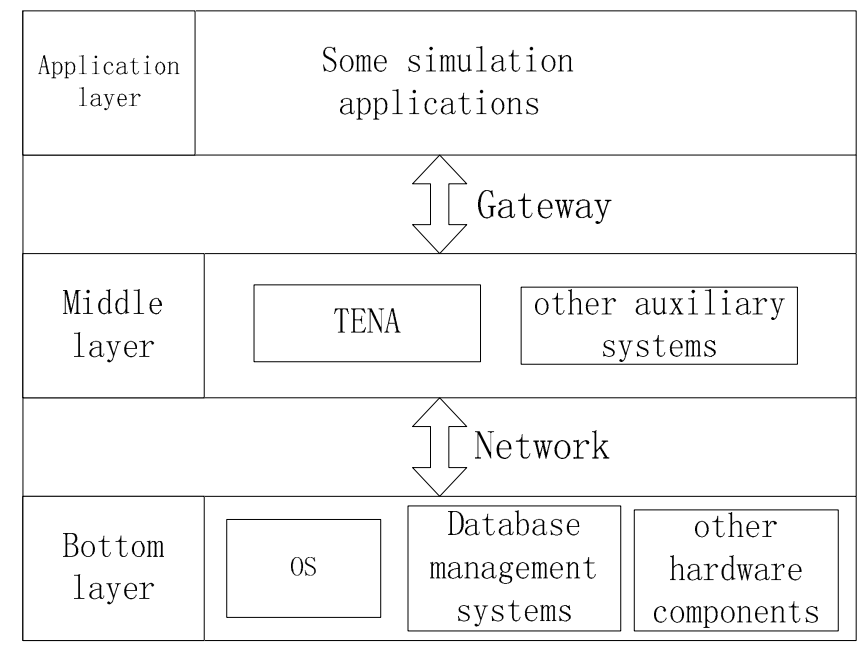

Figure4. virtual range simulation system framework

The bottom layer is the basis of the system. Due to the requirements of security, the bottom layer operating system, database management systems and other infrastructures must be rigorously checked and technological transformed. So these resources must be integrated domestic resources. Because these resources may be located in different regions, so its information through the network communicate with the upper layer.

The middle layer is communication resources based on the TENA. Of course, there are incompatible system resources with TENA. One can increase the functionality of the system, to make up for the lack of TENA; on the other hand can also avoid technical monopoly to develop autonomous virtual simulation system.

Application layer has some simulation software to test and train.They can be input and output, and display the last virtual simulation results. It exchanges information with the middle layer by the gateway .

\section{CONCLUSION}

In this paper, test and training enabling architecture were analyzed, and then analyzes the architecture of TENA, study the application of TENA at last. Summarizing the general framework of virtual simulation system and going on detailed description. TENA is developed by the US. So it is not entirely suitable for our some military needs. However, the basic framework of the TENA that improved virtual firing range simulation system will have to adapt to the characteristics of our army. Improved, both in terms of confidentiality and performance are in line with the status of our army.

\section{REFERENCES}

1. Bo Bi \& Yuanchang Zhu \& Yanqiang Di. 2012. Based Distributed TENA Interactive simulation program design. Microelectronics and Computer: 35-38.

2. Dr. Edward T. Powell \& Dr. J. Russell Noseworthy. The Test and Training Enabling Architecture:10-23.

3. Fansong Meng \& Yong Wang \& Ping Wang. 2009. The Successful Application of TENA architecture in the US JMETC. Modern Defence Technology: 67-72.

4. Jie Zhang.2011. Design of an TENA-Based Distributed VirtualTest System. System Simulation Technology: 58-62.

5. Kun Dai\& Wen Zhao\&Haolong Zhang. 2011.TENA Based Implementation Technology for Virtual Test. Journal of System Simulation: 857-863.

6. Liutao Chen \& Gangyi Ding. 2012. Virtual Target Architecture Design. Journal of Computer Aided Design and Graphics: 1600-1605.

7. Runming Fing\& Guoli Wangi\& Kedi Huang. 2004. Test and Training Enabling Body Studies 
Structure (TENA). Journal of System Simulation: 2280-2284.

8. Runming Fing\& Guoli Wangi\& Kedi Huang.2005. The Comparison of. TENA and HLA. Systems Engineering and Electronics: 288-291.

9. Zhongfu Xu\&Guoli Wang \&Yuzhu Zhang. 2008. TENA Status and Exhibition Hope. System Simulation:6325-6329. 